\title{
1 Genomic divergence, introduction history and latitudinal adaptation of grass carp
}

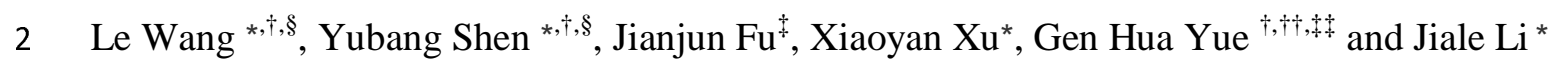

$3{ }^{*}$ Key Laboratory of Exploration and Utilization of Aquatic Genetic Resources, Ministry of

4 Education, Shanghai Ocean University, Shanghai 201306, China

$5 \dagger$ Molecular Population Genetics Group, Temasek Life Sciences Laboratory, 1 Research Link,

6 National University of Singapore, Singapore, 117604, Republic of Singapore

$7 \quad{ }^{\ddagger}$ Key Laboratory of Freshwater Fisheries and Germplasm Resources Utilization, Ministry of

8 Agriculture, Freshwater Fisheries Research Center, Chinese Academy of Fishery Sciences,

$9 \quad$ Wuxi 214081

$10{ }^{\dagger}$ Department of Biological Sciences, National University of Singapore, 14 Science Drive 4,

11 Singapore 117543, Republic of Singapore

$12 \ddagger$ School of Biological Sciences, Nanyang Technological University, 60 Nanyang Drive,

13 Singapore 637551, Republic of Singapore

14 Le Wang and Yubang Shen contributed equally to this study

16 NGS data was deposited in the DDBJ Sequence Read Archive (Project accession no.

17 PRJDB4785). 
22 Keywords: grass carp, genomic divergence, introduction, local selection, latitudinal variation

23 Running title: population genomics of grass carp

25 Corresponding authors

26 Dr. Gen Hua Yue

27 Temasek Life Sciences Laboratory, 1 Research Link, National University of Singapore,

28117604 Singapore

29 Tel: +65-68727405; Fax: +65-68727007; Email: genhua@ tll.org.sg

30 Dr. Jiale Li

31 Key Laboratory of Exploration and Utilization of Aquatic Genetic Resources, Ministry of

32 Education, Shanghai Ocean University, Shanghai 201306, China

33 Tel: +86 021 61900566; FAX: +86 021 61900405; E-mail: jlli2009@ 126.com 


\section{Abstract}

42 Understanding the genomic signatures of population differentiation is fundamental to obtain a 43 comprehensive view of the evolutionary process of organisms. Grass carp is one of the most

44 important fish species in the world due to its significant value in aquaculture and world-wide 45 vegetation biocontrol. However, little is known about the contemporary population structure 46 and also the genetic basis of adaptation to a wide range of latitudinal environments. Using 4743310 SNPs generated by genotyping by sequencing in 197 grass carps from nine 48 populations, we examined the patterns of population differentiation, historical introduction 49 and evidence of local selection. The overall genetic differentiation across all native 50 populations was unexpectedly low. Nevertheless, these native populations were clearly 51 differentiated into three genetic clusters, corresponding to the Yangtze River, the Pearl River 52 and the Heilongjiang River System, respectively. Populations in Malaysia, India and Nepal, 53 with the earliest introduction records, most likely have an origin from the Pearl River System.

54 Using conceptually different approaches, 451 loci were detected under potential local 55 selection, among which 84 were annotated to have a gene feature. $19.0 \%$ of the genes under 56 putative selection were involved in immune responses, while $42.9 \%$ of the annotated loci 57 showed a signature of latitudinal variation. This study provides valuable information for 58 application of genomic tools in addressing questions concerning population differentiation 59 that was influenced by both neutral and adaptive forces, as well as human activities.

\section{Introduction}

61 Grass carp (Ctenopharyngodon idella), belonging to the family Cyprinidae, is a large

62 herbivorous freshwater fish species (Froese and Pauly 2015). It is of great importance as a 63 food fish species as well as a species for world-wide aquatic vegetation control (Cross 1969; 64 Lembi et al. 1978). Grass carp is native to eastern Asia and broadly distributed from the 
65 Heilongjiang River System (Amur River) southward to northern Vietnam (Froese and Pauly

66 2015). According to literature records, grass carp has a culture history of more than 1300

67 years since the Tang Dynasty (FAO 2014). The aquaculture practices are mainly conducted

68 within the geographical regions of the Yangtze and the Pearl River Systems of China (FAO

69 2014). Recent success in artificial breeding has significantly promoted the aquaculture

70 industry of this species (Stanley 1976; Boney et al. 1984; Allen Jr and Wattendorf 1987;

71 Peter et al. 1988). The annual global production has been over 5 million tons since 2013 with

72 an estimated economic value of 5 billion US dollar (FAO 2014). Grass carp is of the highest

73 production yield among all the farmed fish species around the world and accounts for

74 approximately $15.6 \%$ of global freshwater aquaculture production (FAO 2014).

75 Due to herbivorous habits, grass carp has been broadly introduced to more than 40 countries

76 around the world to control the undesirable and/or invasive aquatic plants of freshwater

77 systems (Skelton 2001). Artificial introductions were intensively conducted since the 1960s

78 (Welcomme 1988). The earliest introduction of grass carp was documented from Southern

79 China to Malaysia by Chinese immigrants in the 1800s (Welcomme 1988). However, which

80 native populations these introduced grass carp originated from is unclear. Recently, many

81 studies have reported that introduced/invasive grass carp have endangered native ecological

82 systems and caused great economic loss because they can completely eliminate vegetation

83 from freshwater systems, destroy the populations of native fish species and introduce

84 parasites (Moyle 1986; Chilton II and Muoneke 1992; Bain 1993).

85 Within its native distribution range, grass carp mainly lives in three independent river 86 systems: the Heilongjiang River, the Yangtze River and the Pearl River System (Fu et al.

87 2013). Understanding range-wide population structure is critical to conserve and utilize the 88 genetic resources (Avise 1992). However, it is still not clear, due to a lack of genetic studies 89 (Zhang et al. 2001; Liu et al. 2009; Fu et al. 2013). In particular, grass carp has been cultured 
90 for more than 1300 years (FAO 2014). It is also not known if aquaculture practices with such

91 long history have left significant imprints on the contemporary population structure.

92 Importantly, a geographical pattern of population differentiation is the genetic basis to trace

93 the introduction from native habitats to foreign environments (Cornuet et al. 1999; Paetkau et

94 al. 2004). Investigation on the environments of both native and foreign habitats can provide

95 critical biological information for setting up effective introduction plans (Lande 1988; Bain

96 1993). It can also mitigate the adverse effects on the foreign habitats that were caused by

97 grass carp as an agent of biological invasion (Cross 1969; Bain 1993).

98 Local selection is of critical importance in the evolution of species (Savolainen et al. 2013).

99 Genetic studies focusing on different environments can provide crucial information for understanding the selective forces driving local adaptation (Sultan and Spencer 2002). In some cases, however, local selection acts along certain geographical gradients, e.g. latitude, longitude and altitude, and shows the same direction as the overall neutral forces (Storz 2002; Vasemägi 2006). Thus, it is more challenging to discriminate adaptive evolutionary forces from background neutral forces (McKay and Latta 2002; Storz 2002). Nevertheless, these environmental factors are of great interest in studying the selective forces that shape adaptive divergence (Gilchrist and Partridge 1999; Alberto et al. 2013). Significant associations between environmental variables and genetic markers are typically investigated and considered as footprints of local selection (Sezgin et al. 2004; Vasemägi 2006; Antoniazza et al. 2010). Although adaptive variation can be consistent with "isolation-by-distance" in geographical pattern and also explained by neutral forces like gene flow, genetic drift and admixture between adjacent populations, the footprints of local selection still can be inferred 112 by comparing the relative pattern and strength of population differentiation against environmental variables between candidate loci of adaptive divergence and neutral markers 
115 Grass carp are naturally distributed in a wide range from Southern Siberia to northern 116 Vietnam spanning approximately 30 latitudinal degrees and is tolerant of extreme 117 temperature from $0^{\circ}$ to $38^{\circ} \mathrm{C}$ (Froese and Pauly 2015). The distribution is much likely limited 118 by habitat temperature, and even correlated to the latitudinal variation of temperature. Due to 119 the small number of markers, e.g. microsatellites, however, previous studies only focused on 120 identifying the neutral genetic variations and population structure (Zhang et al. 2001; Liu et 121 al. 2009; Fu et al. 2013). The lack of useful high-density genetic markers limited our understanding on adaptive population differentiation, particularly along latitude (Narum et al. 2013).

Here, a total of 197 grass carp, including both wild populations throughout the whole distribution range and populations with the earliest introduction history were analyzed using ddRAD-Seq approach (Peterson et al. 2012). First, the aim was to identify range-wide population structure and examine the pattern of gene flow, which can help understand the geographic and demographic factors that have played crucial roles in population differentiation of grass carp. Furthermore, we intended to test if the level of population differentiation is sufficient to trace the introduction of grass carp by using the populations with the earliest introduction history. The results can provide useful information for tracing global introduction of grass carp and better utilizing this species in the biocontrol of aquatic vegetation. In addition, as the native populations show a significant latitudinal distribution pattern, our goal was to detect the footprints of local selection and discuss if such divergence was correlated to specific environmental factors, particularly temperature. Finally, we aimed to identify candidate genes under potential directional selection, which help understand the mechanism of evolution under selection. In total, this study can disentangle the effects of demographic history, gene flow and local selection on the contemporary population 
140 biocontrol and understanding the adaptive divergence of freshwater fish species in the

141 presence of complicated gene flow and demographic history.

\section{Materials and Methods}

\section{Sampling and data collection}

144 Grass carp including six wild and three introduced populations consisting of 197 individuals 145 were collected between 2007 and 2008. The wild populations were from the three river 146 systems: the Heilongjiang River, the Yangtze River and the Pearl River System, across this 147 species' distribution range, while the three introduced populations were sampled from 148 Malaysia, India and Nepal, respectively (Table 1 \& Figure 1). The annual average 149 temperature of each sampling site was retrieved from weather.sina.com.cn (Table 1). 150 Population Malaysia was documented as being introduced in the1800s from southern China, 151 while population India was recorded as being introduced from Hong Kong, China (the Pearl 152 River System), in 1959 and 1968 (Welcomme 1988). Population Nepal was set up by 153 introduction from India in 1966-1967 (Shireman and Smith 1983). All samples were 154 estimated as more than one year old. Fin tissue was collected and preserved in $95 \%$ ethanol at $155-20^{\circ} \mathrm{C}$. Genomic DNA was isolated using DNeasy Blood \& Tissue Kit (Qiagen, Germany) 156 and quantified using Qubit ${ }^{\circledR}$ assays (Life Technologies, USA).

\section{Genotyping by sequencing}

158 Genotyping by sequencing (GBS) was conducted using the ddRAD-Seq approach (Peterson 159 et al. 2012). Restriction enzymes PstI-HF and MspI (New England Biolabs, USA) were 160 selected for library construction. $200 \mathrm{ng}$ genomic DNA was fully digested with two enzymes. 161 Digested fragments were ligated with barcoded adaptors using T4 ligase (New England 162 Biolabs, USA) and then pooled for cleanup with QIAquick PCR Purification Kit (Qiagen, 
163 Germany). The cleaned products were size selected and purified (300-500 bp) by running

164 gels and using QIAquick Gel Extraction Kit (Qiagen, Germany), respectively. The recovered

165 libraries were then amplified using Phusion® High-Fidelity DNA Polymerase (New England

166 Biolabs, USA). After a final cleanup using QIAquick PCR Purification Kit (Qiagen,

167 Germany), the libraries were sent to a NextSeq 500 platform (Illumina, USA) for 2x150 bp

168 paired-end sequencing.

169 The program process_radtags (Catchen et al. 2011) was employed to filter the raw 170 sequencing reads with default parameters and reads with any uncalled base were removed.

171 Clean reads were then demultiplexed and trimmed to $100 \mathrm{bp}$ for in silico mapping. First,

172 reads were mapped to the reference genome of grass carp v1.0 (Wang et al. 2015b) using the

173 program BWA-MEM with default parameters (Li and Durbin 2010). Reads with multiple

174 targets in the reference were excluded from further analysis. Reference-aligned reads were

175 then assembled into stacks for each individual using pstacks implemented in the package

176 Stacks v1.34 (Catchen et al. 2011). A total of 54 individuals randomly selected from each

177 population were used to construct a catalogue of stacks using cstacks. Stacks from each

178 individual were then matched against the catalogue for SNP discovery using sstacks. Finally,

179 genotyping was conducted across all populations using the program populations with a

180 minimum of $10 \times$ sequence depth. SNPs were further filtered to meet the following criteria:

181 present in $>70 \%$ of the individuals in each population, have no more than two alleles and

182 show an observed heterozygosity of $<0.5$ (Hohenlohe et al. 2010). Only one SNP was

183 retained for each RAD locus. Hardy-Weinberg equilibrium (HWE) for each locus was

184 examined using Genepop v4.2 (Raymond and Rousset 1995) and loci that deviated from

185 HWE in a single population at the significance level of 0.01 were excluded from further 186 analysis.

\section{Population structure and phylogenetic relationship}


188 Genetic diversity for each population was measured by observed heterozygosity $\left(H_{O}\right)$, expected heterozygosity $\left(H_{E}\right)$ and nucleotide diversity $(\Pi)$, while genetic divergence for each individual locus was estimated using F-statistics (Weir and Cockerham 1984). All these calculations were performed using the program populations (Catchen et al. 2011). Population genetic divergence was estimated in the form of pairwise $F_{S T}$ using the program Arlequin 3.5 (Excoffier and Lischer 2010). Statistical significance was examined using an exact test with 10000 permutations. Population structure at both the population and individual levels was investigated by principle component analysis using the program Eigenstrat v5.1 (Price et al. 2006). The pattern of population differentiation was examined in the form of isolation-bydistance (IBD) using Mantel tests with the program IBD v1.52 (Bohonak 2002). The genetic distance was measured using $F_{S T} / 1-F_{S T}$, while the geographical distance was estimated as the linear distance between sampling localities. approach with the program Populations v1.2.33 (Langella 1999) by bootstrapping over loci for 1000 times. The origins of introduced populations from native populations were determined using ancestral alleles. The software fastStructure v1.0 (Raj et al. 2014) was employed to infer the ancestral alleles between one introduced population and two native populations. The two selected native populations were from the Yangtze River and the Pearl River systems, respectively, and had the closest phylogenetic relationship with the introduced population. The program was run 10 times for each $\mathrm{K}$ value (from 1 to 6 ) with default parameters. The most likely number of genetic clusters $(\mathrm{K})$ was estimated by plotting the marginal likelihood value. 
211 In order to identify evidence of latitudinal variation at SNP loci, we independently estimated

212 the association between allele frequencies for each SNP and latitude at the population level

213 using a liner correlation method. Pairwise genetic distance based on allele frequencies of

214 individual locus was estimated according to the method by Reynolds et al. (1983). Genetic

215 distance was then correlated to geographical distance among populations using Mantel tests

216 to discriminate neutral mutations from the loci showing latitudinal variation in allele

217 frequencies. By removing the loci under putative neutral processes, a set of candidate SNPs

218 showing latitudinal variation but not isolation-by-distance in genetic divergence was obtained.

219 Evidence of local adaptation was detected for individual locus using a Bayesian generalized

220 linear mixed model involving covariance of allele frequencies and environmental variables

221 with the program Bayenv (Coop et al. 2010). A Bayes factor (BF) was calculated for each

222 SNP to measure the strength of the correlation between SNP variation and environmental

223 variables. According to the method by Coop et al. (2010), a BF > 3 was considered as a

224 substantial evidence for selection. The program was run for five times with an independent

225 variance-covariance matrix of population genetic variation to achieve consistency among the

226 runs.

$227 F_{S T}$-based outlier tests were also performed to identify signatures of spatial purifying

228 selection. Outlier loci under directional selection are expected to show higher levels of

229 divergence, while loci under balancing selection would show lower levels of genetic

230 divergence compared to the putative neutral loci (Beaumont and Nichols 1996; Foll and

231 Gaggiotti 2008). Firstly, a Bayesian simulation-based test implemented in BayeScan (Foll

232 and Gaggiotti 2008) was used to identify outlier SNPs. Loci with Bayes factor > 3 were

233 considered as substantial outliers. Considering that grass carp are distributed in different river

234 systems and that there is among-group genetic structure, a hierarchical island model

235 (Beaumont and Nichols 1996) for identifying outlier loci was also employed using the 
236 program Arlequin 3.5 (Excoffier and Lischer 2010) with the following parameters: 50000

237 simulations, 10 simulated groups, and 100 demes per group. Only outliers above the $99 \%$

238 quantile of the null distribution were considered as candidates under spatial purifying 239 selection.

240 Analysis of the genes under putative selection

241 Loci under putative directional selection were functionally annotated by Blast2Go (Conesa et 242 al. 2005) against all available nucleotide databases with an E-value cutoff of $10^{-6}$. SNPs 243 within both exons and introns were considered to have a gene feature. Enrichment of Gene 244 Ontology (GO) terms was conducted using the program WEGO (Ye et al. 2006) with default 245 parameters. Loci were also mapped to the reference genome of zebrafish (GRCz10) using 246 Blastx to retrieve the corresponding Ensembl gene IDs. A more detailed functional 247 annotation of these genes were then performed by mapping to the Kyoto Encyclopedia of 248 Genes and Genomes (KEGG) pathway database (Kanehisa and Goto 2000) using the program 249 David (Huang et al. 2009). The signaling pathways of at least two genes in default were 250 enriched for further analysis.

251 Results

252 SNP discovery and genotyping

253 In total, NGS produced an average of 10.17 million raw reads for each individual. After 254 quality control, 9.21 million reads per individual were obtained for sequence mapping and 255 SNP discovery. A total of 280544 SNPs were discovered across all nine populations. After 256 removing the loci that failed to meet the filtering criteria, 43310 SNPs were genotyped across 257 all populations, among which 35844 (82.8\%) showed minor allele frequency (MAF) of > 2580.05. 


\section{Genetic diversity and population structure}

260 The measures of genetic diversity, including $H_{O}, H_{E}$ and $\Pi$, estimated based on all variant

261 SNPs were shown in Table 1. For the six wild populations, Vietnam showed slightly lower 262 genetic diversity than the others. We also observed that the genetic diversity of the introduced 263 populations including Malaysia, India and Nepal were significantly lower than that of the wild populations $(P<0.001$, one-way ANOVA test).

265 Pairwise $F_{S T}$ analysis revealed significant genetic differentiation between the wild populations and the introduced populations with $F_{S T}$ ranging from 0.1126 between Zhaoqing and India to 0.2399 between Vietnam and Malaysia (Table 2). However, genetic differentiation between each pair of wild populations was shallow with $F_{S T}$ ranging from 0.0073 between Jiujiang and Shishou to 0.0515 between Hanjiang and Vietnam, although significantly different from 0 . The wild population Zhaoqing from the Pearl River System showed slightly lower genetic differentiation with the three introduced populations: Malaysia, India and Nepal, compared to the other wild populations $(\mathrm{P}<0.05$, paired $t$-test $)$. For wild populations, genetic divergence at individual locus was estimated between the most divergent populations (Hanjiang vs Vietnam) and also between the most distant populations (Nenjiang vs Vietnam). We observed that no loci showed $F_{S T}>0.5$ and only $<15 \%$ of loci had $F_{S T}>$ 0.1 for both population pairs (Figure 2). Loci showing $F_{S T}>0.1$ were found more frequently between Nenjiang and Vietnam (14.5\%) than between Hanjiang and Vietnam (5.6\%).

278 Principle component analysis revealed that the wild populations were strikingly differentiated 279 from the introduced populations (Figure 3a). For the wild populations, Zhaoqing and Vietnam from the Pearl River System were clearly differentiated from the populations from both the Yangtze River System (Hanjiang, Jiujiang and Shishou) and the Heilongjiang River System (Nenjiang), although there was some mixture of individuals between the Yangtze

283 River and the Heilongjiang River Systems (Figure 3b). The pattern of genetic differentiation 
284 across all wild populations rejected the model of isolation-by-distance $\left(R^{2}=0.187, P=0.16\right.$;

285 Figure 4a). Considering that some individuals of the Heilongjiang River System very likely

286 have an origin from the Yangtze River System (Figure 3b), the population Nenjiang was

287 further removed from Mantel tests. Interestingly, we identified a strong correlation between

288 population differentiation and geographical distance $\left(R^{2}=0.876, P<0.001\right.$; Figure 4b).

289

290

291

292

293

294

\section{Population introduction history}

The phylogenetic tree showed that native populations from the Yangtze River (Hanjiang, Jiujiang and Shishou), the Heilongjiang River (Nenjiang) and the Pearl River (Zhaoqing and Vietnam) Systems formed three independent clusters, respectively, with the Heilongjiang River System cluster located between the Yangtze River System and the Pearl River System clusters (Figure 5a). For the introduced populations, India and Nepal formed one subcluster and joined into the Pearl River System cluster. Although joined into the Pearl River System, the introduced population Malaysia showed a relatively long genetic distance with the other populations within this cluster and was relatively close to the Heilongjiang River System. Considering the results of the principle component analysis, population Nenjiang and Malaysia might have an origin of admixture between the Yangtze River and the Pearl River System lineages (Figure 5a). We further inferred the origin of the two populations separately using ancestral alleles with the program fastStructure. Two populations of the closest genetic relationship, Jiujiang and Zhaoqing from the Yangtze River and the Pearl River System, respectively, were selected as the potential ancestral populations. Considering most of the SNPs have a very low level of genetic divergence (Figure 2), only loci of $F_{S T}>0.05$, numbering 5986, were used for these analyses. The most likely $\mathrm{K}$ values for estimation of the origins of the populations Nenjiang and Malaysia were inferred as 2 and 3, respectively (online supporting Figure S1). We found that many more ancestral alleles in the Nenjiang population in the Heilongjiang River System originated from the Yangtze River System than 
309 from the Pearl River System (Figure 5b). However, for the introduced population Malaysia, 310 only ancestral alleles from the Pearl River System (Zhaoqing) were observed (Figure 5c).

\section{Identifying loci under putative selection}

312 Among all the SNPs with MAF > 0.05, 5197 (14.4\%) were found to have significant linear 313 regression $(\mathrm{P}<0.05)$ between latitudinal gradients and allele frequencies. Mantel tests 314 revealed that $3351(9.4 \%)$ SNPs showed significant patterns of isolation-by-distance $\left(R^{2}>\right.$ $3150.514, P<0.05)$ for individual locus. As stated above, the Nenjiang population showed a signature of admixture, and this produced decisive effects on the pattern of isolation-bydistance (Figure 3 \& Figure 4). Therefore, we further removed this population from Mantel tests. Using five native populations, 3710 (10.4\%) SNPs were observed to have significant patterns of isolation-by-distance $\left(R^{2}>0.632, P<0.05\right)$. After removing loci with any evidence of isolation-by-distance, a total of 2700 loci that showed significant latitudinal variation in allele frequencies were obtained for further analysis (Figure 6).

The Bayesian generalized linear mixed model identified 768 SNPs that showed significant association between genetic variations and latitudinal gradients across all six populations at individual locus $(\mathrm{BF}>3)$. In $F_{S T}$ based outlier tests, BayeScan detected 263 SNPs as substantial outliers (BF > 3), while the hierarchical island model identified 744 SNPs as significant outliers at the significance level of 0.99. A total of $791(2.2 \%)$ unique loci were revealed to be outliers by the two $F_{S T}$ based tests.

Within the 2700 loci that showed correlation to latitudinal gradients, 132 were candidates under directional selection as revealed by the program Bayenv and both $F_{S T}$ based outlier tests (Figure 6). Moreover, Bayenv and the outlier tests identified 265 and 285 unique candidates under spatially purifying selection, respectively (Figure 6). We observed that the loci under putative selection revealed by the two outlier tests and Bayenv had much higher 
333 genetic divergence with mean $F_{S T}$ values of 0.198 and 0.104 , respectively. However, the loci

334 correlated to latitudinal gradients showed a much lower mean $F_{S T}(0.034)$ than the loci with

335 the pattern of $\operatorname{IBD}\left(F_{S T}, 0.065\right)$ and also the whole dataset $\left(F_{S T}, 0.037\right)$ (Figure 7). Low

336 genetic divergence much likely suggests that selection pressure is weak on these loci. In order

337 to reduce false positives, only loci that were revealed to be under potentially directional

338 selection by outlier tests, Bayenv or allele frequencies association study, and also showed $F_{S T}$

339 of more than the $95 \%$ quantile $(0.121)$ of the whole dataset, were retained, which produced

340451 loci for further analyses.

\section{Functional annotation of genes under putative selection}

342 Among the 451 loci, $84(18.6 \%)$ were annotated as having a gene feature and were further

343 investigated (Table S1). $42.9 \%$ (36) of the annotated genes were indicated to be significantly

344 associated with latitudinal gradients as revealed by Bayenv or allele frequency correlation

345 study. GO enrichment revealed that these genes covered a wide range of functions in

346 biological processes: biological regulation, cellular process, developmental process, immune

347 system process, metabolic process, pigmentation and response to stimulus (online

348 supporting Figure S2). Three KEGG pathways: Focal adhesion, Vascular smooth muscle

349 contraction and the Toll-like receptor signaling pathway, were enriched with each containing

350 two genes (Table S2). Interestingly, by searching for literature, we found 16 (19.0\%) genes

351 that play important roles in immune responses (e.g. MHC I UAK and MHC II DAB) and/or

352 antiviral responses (e.g. Myxovirus resistance proteins and Mitochondrial antiviral signalling

353 protein) (Table 3). Among these immune-related genes, $8(50.0 \%)$ showed a pattern of

354 latitudinal variation as revealed by Bayenv or allele frequency correlation study, while the

355 other 8 genes were suggested to be under spatially purifying selection (Table 3 ).

\section{Discussion}


357 In this study, we investigated range-wide population structure of native populations and origin of introduced populations in the South and Southeast Asia, as well as latitudinal variation and local selection of grass carp using population genomic approaches. This study provides important implications for future application of grass carp in the biocontrol of aquatic vegetation and understanding the mechanism of local adaptation, particularly adaptive latitudinal variation for freshwater fish species with a wide range of geographical distribution.

\section{High gene flow among native populations}

Typically, freshwater fishes are more isolated by various geographical factors than marine fishes and thus have a lower level of gene flow (Ward et al. 1994). In this study, we observed that pairwise population genetic differentiation was very low $\left(F_{S T}, 0.0073-0.0515\right)$, comparable to a previous study using microsatellites (Liu et al. 2009). This level of gene flow across the native populations of grass carp is much higher than the average for freshwater species (Gyllensten 1985; Ward et al. 1994; Cooke et al. 2012) and even higher than some fishes living in the open marine environment, e.g. the Chinook salmon (Oncorhynchus tshawytscha) (Larson et al. 2014), Atlantic salmon (Salmo salar) (Bourret et al. 2013) and Asian seabass (Lates calcarifer) (Wang et al. 2015a). Further investigation on genetic differentiation at individual locus revealed that only $<15 \%$ of total loci showed $F_{S T}>0.1$ and no loci had $F_{S T}>0.5$. Considering the geographical isolation among the three river systems, such results are rather unexpected. The genetic distance between the Pearl River System (Vietnam) and the Yangtze River System (Hanjiang) was closer than between the Pearl River System (Vietnam) and the Heilongjiang River System (Nenjiang), although the latter showed a rather longer geographical distance. Nevertheless, we observed that the number of loci with $F_{S T}>0.1$ was much more between Nenjiang and Vietnam than between 
these contradicting results strongly suggest that the high gene flow among the three river systems is not only naturally occurring but also induced by human activities. Interestingly, the observed pattern of population differentiation did not conform to isolation-by-distance across the whole data set. However, after removal of the Nenjiang population that was suggested to have an admixture origin between the Yangtze River and the Pearl River Systems, the remaining populations showed a strong signal of isolation-by-distance. This indicates that although human-induced gene flow might have played important roles in shaping the overall population structure of grass carp, it only showed overwhelming importance in the Heilongjiang River System.

According to historical records, grass carp was abundant in both the Yangtze River System and the Pearl River System, and was widely captured from the wild as seeds for aquaculture locally (FAO 2014). There was no practical need to introduce grass carp between the two river systems. On the other hand, it is reasonable that gene flow can be high between the two river systems because they partially overlap in geography (Zhu 1993). For these reasons, the migration occurred much more naturally and thus the population differentiation showed a strong pattern of isolation-by-distance. However, we cannot exclude the possibility that human activities played important roles in dispersal of grass carp. Such gene flow might be merely induced so randomly with no directional purpose that it has much less effects on shaping genetic structure than the natural gene flow.

In contrast to the Yangtze and the Pearl River systems, the distribution and culture of grass carp in the Heilongjiang River System have never been abundant nor considered as a major aquaculture practice according to both historical records and current official fishery statistics (FAO 2014; Liu and Li 2015). Geographically, the Heilongjiang River System is completely isolated with the other two river systems. On the other hand, the Nenjiang population was mixed with alleles originating from both the Yangtze River and the Pearl River Systems as 
407 inferred from fastStructure. Hence, the low genetic differentiation between this river system

408 and the other river systems strongly indicates that human-induced dispersal played more

409 important roles than natural introgression. In fact, grass carp in the Heilongjiang River

410 System grow slower than in the other river systems due to low water temperature (Cui et al.

411 1995). Therefore, seeds from the other river systems, particularly from the Yangtze River

412 system, were commonly introduced for aquaculture purposes because of geographical

413 adjacency. This is the most likely explanation for the low genetic differentiation of grass carp

414 between the Heilongjiang River System and the other river systems.

\section{Origins of the early introduced populations}

416 Both in terms of genetic diversity and differentiation, we observed significant genetic

417 heterogeneity between all of the native populations and the introduced populations including

418 Malaysia, India and Nepal, suggesting significant founder effects in the introduction of these

419 populations (Barton and Charlesworth 1984). It is likely that the introduction of grass carp

420 was not initiated under planned programs or that not all the introduced fish can adapt to the

421 new habitats. Genetic differentiation among the native populations is the basis to trace the

422 origins of the introduced populations (Cornuet et al. 1999; Paetkau et al. 2004). As expected,

423 we identified significant genetic differentiation and also a clear geographical pattern of

424 population differentiation among the three river systems in a background of high gene flow.

425 Native populations from the Heilongjiang River, the Yangtze River and the Pear River

426 Systems were separately clustered into independent genetic lineages, although there was

427 evidence of population admixture for the Heilongjiang River System. These results provided

428 critical clues to trace back population origins. Both pairwise $F_{S T}$ and phylogenetic analyses

429 indicated that all three introduced populations, Malaysia, India and Nepal originated from the

430 Pearl River System, which was also supported by the data inferred from ancestral alleles. 
431 First, the Pearl River System is geographically more adjacent to Malaysia, India and Nepal 432 than the Yangtze River and the Heilongjiang River Systems. Therefore, it is reasonable that 433 the Pearl River System was preferred as the source for introduction to these countries. In 434 contrast, the Yangtze River and the Heilongjiang River Systems are not only distant from 435 Southeast and the South Asia, but also isolated by various continental barriers, e.g. the 436 Himalaya Mountains. It is a great challenge to introduce fish from these two river systems to 437 Southeast and the South Asia. Most importantly, it was recorded that grass carp was first 438 introduced into Malaysia from Southern China with the large-scale migration of Chinese 439 people in the 1800 s, although it is not clear which river system the Malaysia population 440 originated from (Welcomme 1988). The introduction history could be inferred from the 441 routes of Chinese migration during that time. As revealed by history studies, most of the 442 Chinese people in Southeast Asia were from Guangdong and Fujian provinces (Pan 1999), 443 which geographically overlap with the Pearl River System. Thus, the Malaysia population 444 very likely has an origin in the Pearl River System, consistent with the results of the genetic 445 data.

446 In total, our data suggest that the native populations might have accumulated enough genetic 447 divergence for population origin assignment of the recently introduced populations of grass 448 carp, e.g. the populations introduced to Europe, North America and also some Southern 449 Hemisphere countries (Mitchell 1986). These results are very valuable for studying the 450 production and physiological adaptation, as well as the living environments and habitat 451 preferences, of both native and introduced populations. Such information can be referenced to 452 construct comprehensive introduction plans in the future.

\section{Local selection and latitudinal variation}


454 It is a great challenge to discriminate local selection from neutral processes for organisms that

455 have experienced complicated demographic history. Neutral processes can generate the same 456 marks on genomic architecture as local selection does (Storz 2002; Vasemägi 2006;

457 Savolainen et al. 2011; Wang et al. 2013; McKown et al. 2014; Hornoy et al. 2015). In some

458 cases, adaptive traits show a specific distribution pattern along specific environmental factors.

459 If the estimates of neutral forces are coincidentally varying along the same environmental

460 factors, the difficulty of disentangling the roles of adaptive driving forces would be greatly

461 enhanced (Merilä and Crnokrak 2001; McKay and Latta 2002; Storz 2002). Under this

462 condition, a single association test between an individual locus and an environmental factor is

463 obviously not enough to determine if one locus has experienced spatially divergent selection,

464 particularly in the background of genome-wide patterns of isolation-by-distance (Vasemägi

465 2006). Grass carp is such a species, which has a significant signature of latitudinal

466 distribution. Thus, the adaptive traits might vary in parallel with the pattern of neutral

467 processes along specific geographical gradients, like latitude. These evolutionary processes

468 limited the potential to identify the molecular mechanism underlying adaptive evolution

469 (McKay and Latta 2002; Chen et al. 2012). Here, we used conceptually different approaches

470 to differentiate the footprints of local selection from the currents of neutral evolutionary

471 processes (Hansen et al. 2010; Wang et al. 2013).

472 Our main purpose was to identify individual loci of higher genetic divergence than can be

473 explained by random genetic drift and gene flow (Storz 2002). As discussed above, grass carp

474 from different river systems very likely have unique demographic history. Grass carp

475 originated from the Yangtze River System and expanded into the Pearl River and the

476 Heilongjiang River Systems during the Pleistocene and Pliocene, respectively (Li and Fang

477 1990). Nevertheless, the contemporary population structure was significantly shaped by high

478 levels of gene flow due to both natural and artificial factors. As the Pearl River System and 
479 the Heilongjiang River System cover the southernmost and the northernmost distribution 480 ranges, respectively, such contrasting environments have likely posed strong selective 481 pressure on the distribution of grass carp (Gardner and Latta 2006). Gene flow within the 482 Pearl River and the Yangtze River Systems might be seldom influenced by human activities, 483 as population differentiation still shows a significant pattern of isolation-by-distance. 484 However, gene flow between the Heilongjiang River System and the other two river systems 485 were profoundly influenced by recent human activities, which overall changed the 486 geographical pattern of population differentiation such that the pattern of isolation-by487 distance was no longer observed. Although influenced by human activities, the extreme 488 northernmost environmental condition of the Heilongjiang River System can pose strong 489 selective pressure on the introduced grass carp. Such a process of natural selection provides 490 important clues to discriminate footprints of natural selection from genome-wide patterns of 491 isolation-by-distance.

492 Consistent with the overall neutral evolutionary process, a large number of loci, 6489 (18.1\%) 493 of the total loci, were indicated to show a pattern of isolation-by-distance in genetic 494 divergence. Although 14.4\% (5197) were revealed to have significant correlations between 495 latitudinal gradients and allele frequencies, some of them would be false positives because 496 these loci also showed significant correlations between pairwise geographical distance and 497 genetic divergence. In total, these results suggest a strong background of isolation-by498 distance in the overall population differentiation of grass carp. After removing the loci which 499 were potential false positives by application of a series of conceptually different approaches, 500 only 451 loci were suggested to be under putative directional selection, accounting for $1.3 \%$ 501 of the total loci. This ratio is much less than in previous studies $(2.3 \%-10 \%)$ using fish 502 species that showed weak or non-significant genome-wide patterns of isolation-by-distance, 503 like Atlantic salmon (Bourret et al. 2013), Chinook salmon (Larson et al. 2014) and 
504 yellowfin tuna (Thunnus albacares) (Grewe et al. 2015). Such results likely suggest that it is 505 much less efficient to identify loci under putative directional selection with a genome-wide 506 pattern of isolation-by-distance (Beaumont and Nichols 1996). Among the loci under putative 507 directional selection, $18.6 \%$ were revealed to be associated with functional genes. 508 Interestingly, $42.9 \%$ (36) of the annotated genes were indicated to be significantly correlated 509 to latitudinal gradients, indicating clinally adaptive divergence at these loci (Storz 2002; 510 Vasemägi 2006; Chen et al. 2012). Although these genes are involved in various functions, 511 we observed a significant cluster of genes $(16,19.0 \%)$ playing important roles in immune responses (e.g. MHC I UAK and MHC II DAB) (Benacerraf 1981) and/or antiviral responses (e.g. Myxovirus resistance proteins and Mitochondrial antiviral signalling protein) (Seth et al. 2005; Gao et al. 2011). Among these immune-related genes, 8 (50.0\%) showed a pattern of latitudinal adaptive variation. This result further suggests that the distribution of grass carp spanning approximately 30 latitudinal degrees was also the consequence of clinal adaptation along latitude. In the case of grass carp, the annual average temperature was observed to be highly correlated to the latitude of the sampling sites $\left(R^{2}=0.992, P<0.001\right)$. However, we did not find any evidence that the enriched genes were associated to thermal adaptation. This result might suggest that the latitudinal adaptive distribution of grass carp was not directly selected by the temperature. Because the enriched genes were observed to have functions in defense against various pathogens and the diversity of pathogens were strongly related to the

523 environmental temperature (Cashdan 2001; Mitchell et al. 2005; Dionne et al. 2007), our 524 results might suggest that the latitudinal adaptation or clinal adaption of grass carp along 525 latitude was the consequence of selection by pathogens and indirectly by temperature of the 526 habitats.

In total, the joint application of different approaches identified a promising set of loci that were under putative directional selection. Many of them have a pattern of latitudinal 
529 variations. The latitudinal distribution of grass carp likely has an adaptive genetic basis,

530 although the underlying causes remain to be elucidated. Nevertheless, spatially purifying

531 selection has played important roles in shaping the contemporary population structure of

532 grass carp. Our data shed light on the genetic basis of local adaptation of grass carp with a

533 large distribution range.

\section{Acknowledgements}

535 We thank Mr. Narayan Prasad Pandit and Mr. Dang Hai Nguyen for sample collection from 536 Vietnam, Indian and Nepal respectively. This research is supported by the National Key 537 Technology R\&D Program of China (2012BAD26B02) and the China Agriculture Research 538 System (CARS-46-04) foundations.

\section{Authors' contributions}

540 YS, LW, JL and GHY conceived the study and finalized the manuscript. YS and LW 541 designed the experiments. YS, JF and XX carried out the lab experiments. LW and YS 542 performed bioinformatics, analysed the molecular data and drafted the manuscript. All 543 authors have read and approved the final manuscript.

\section{$544 \quad$ References}

545 Alberto, F. J., J. Derory, C. Boury, J.-M. Frigerio, N. E. Zimmermann et al., 2013 Imprints of 546 natural selection along environmental gradients in phenology-related genes of $547 \quad$ Quercus petraea. Genetics 195: 495-512.

548 Allen Jr, S. K., and R. J. Wattendorf, 1987 Triploid grass carp: status and management 549 implications. Fisheries 12: 20-24. 
550 Antoniazza, S., R. Burri, L. Fumagalli, J. Goudet and A. Roulin, 2010 Local adaptation maintains clinal variation in melanin-based coloration of European barn owls (Tyto alba). Evolution 64: 1944-1954.

Avise, J. C., 1992 Molecular population structure and the biogeographic history of a regional fauna: a case history with lessons for conservation biology. Oikos 63: 62-76.

Bain, M. B., 1993 Assessing impacts of introduced aquatic species: grass carp in large systems. Environmental Management 17:211-224.

Barton, N. H., and B. Charlesworth, 1984 Genetic revolutions, founder effects, and speciation. Annual Review of Ecology and Systematics 15: 133-164.

Beaumont, M. A., and R. A. Nichols, 1996 Evaluating loci for use in the genetic analysis of population structure. Proceedings of the Royal Society of London B: Biological Sciences 263: 1619-1626.

Benacerraf, B., 1981 Role of MHC gene products in immune regulation. Science 212: 12291238.

Bohonak, A., 2002 IBD (isolation by distance): a program for analyses of isolation by distance. Journal of Heredity 93: 153-154.

Boney, S. E., W. L. Shelton, S.-L. Yang and L. Wilken, 1984 Sex reversal and breeding of grass carp. Transactions of the American Fisheries Society 113: 348-353.

Bourret, V., M. P. Kent, C. R. Primmer, A. Vasemägi, S. Karlsson et al., 2013 SNP-array reveals genome-wide patterns of geographical and potential adaptive divergence across the natural range of Atlantic salmon (Salmo salar). Molecular Ecology 22: $532-551$.

Cashdan, E., 2001 Ethnic diversity and its environmental determinants: Effects of climate, pathogens, and habitat diversity. American Anthropologist 103: 968-991. 
574 Catchen, J. M., A. Amores, P. Hohenlohe, W. Cresko and J. H. Postlethwait, 2011 Stacks:

575 building and genotyping loci de novo from short-read sequences. G3: Genes,

$576 \quad$ Genomes, Genetics 1: 171-182.

577 Chen, J., T. Källman, X. Ma, N. Gyllenstrand, G. Zaina et al., 2012 Disentangling the roles of

578 history and local selection in shaping clinal variation of allele frequencies and gene

$579 \quad$ expression in Norway spruce (Picea abies). Genetics 191: 865-881.

580 Chilton II, E. W., and M. I. Muoneke, 1992 Biology and management of grass carp

581 (Ctenopharyngodon idella, Cyprinidae) for vegetation control: a North American

582 perspective. Reviews in Fish Biology and Fisheries 2: 283-320.

583 Conesa, A., S. Götz, J. M. García-Gómez, J. Terol, M. Talón et al., 2005 Blast2GO: a

584 universal tool for annotation, visualization and analysis in functional genomics

585 research. Bioinformatics 21:3674-3676.

586 Cooke, G. M., N. L. Chao and L. B. Beheregaray, 2012 Divergent natural selection with gene

587 flow along major environmental gradients in Amazonia: insights from genome scans,

588 population genetics and phylogeography of the characin fish Triportheus albus.

$589 \quad$ Molecular Ecology 21: 2410-2427.

590 Coop, G., D. Witonsky, A. Di Rienzo and J. K. Pritchard, 2010 Using environmental

591 correlations to identify loci underlying local adaptation. Genetics 185: 1411-1423.

592 Cornuet, J.-M., S. Piry, G. Luikart, A. Estoup and M. Solignac, 1999 New methods

593 employing multilocus genotypes to select or exclude populations as origins of

$594 \quad$ individuals. Genetics 153: 1989-2000.

595 Cross, D., 1969 Aquatic weed control using grass carp. Journal of Fish Biology 1: 27-30.

596 Cui, Y., S. Chen and S. Wang, 1995 Effect of temperature on the energy budget of the grass

597 carp, Ctenopharyngodon idellus. Oceanologia et Limnologia Sinica 26: 169-174. 
598 Dionne, M., K. M. Miller, J. J. Dodson, F. Caron and L. Bernatchez, 2007 Clinal variation in

599 MHC diversity with temperature: evidence for the role of host-pathogen interaction

600 on local adaptation in Atlantic salmon. Evolution 61: 2154-2164.

601 Excoffier, L., and H. E. Lischer, 2010 Arlequin suite ver 3.5: a new series of programs to 602 perform population genetics analyses under Linux and Windows. Molecular Ecology

$603 \quad$ Resources 10: 564-567.

604 FAO, 2014 Cultured Aquatic Species Information Programme Ctenopharyngodon idellus,

605 Rome, www.fao.org/fishery/culturedspecies/Ctenopharyngodon_idellus, (accessed

$606 \quad 10 / 2015)$

607 Foll, M., and O. Gaggiotti, 2008 A genome-scan method to identify selected loci appropriate

608 for both dominant and codominant markers: a Bayesian perspective. Genetics 180:

$609 \quad 977-993$.

610 Froese, R., and D. Pauly, 2015 FishBase. World Wide Web electronic publication,

611 www.fishbase.org, (accessed 10/2015).

612 Fu, J., J. Li, Y. Shen, R. Wang, Y. Xuan et al., 2013 Genetic variation analysis of wild populations of grass carp (Ctenopharyngodon idella) using microsatellite markers. Hereditas 35: 192-201.

615 Gao, S., A. von der Malsburg, A. Dick, K. Faelber, G. F. Schröder et al., 2011 Structure of 616 myxovirus resistance protein a reveals intra-and intermolecular domain interactions 617 required for the antiviral function. Immunity 35: 514-525.

618 Gardner, K. M., and R. G. Latta, 2006 Identifying loci under selection across contrasting 619 environments in Avena barbata using quantitative trait locus mapping. Molecular Ecology 15: 1321-1333. 
621 Gilchrist, A. S., and L. Partridge, 1999 A comparison of the genetic basis of wing size divergence in three parallel body size clines of Drosophila melanogaster. Genetics 153: $1775-1787$. Acids Research 28: 27-30.

Grewe, P., P. Feutry, P. Hill, R. Gunasekera, K. Schaefer et al., 2015 Evidence of discrete yellowfin tuna (Thunnus albacares) populations demands rethink of management for this globally important resource. Scientific Reports 5: 16916.

Gyllensten, U., 1985 The genetic structure of fish: differences in the intraspecific distribution of biochemical genetic variation between marine, anadromous, and freshwater species. Journal of Fish Biology 26: 691-699.

Hansen, M. M., K. Meier and K. L. D. Mensberg, 2010 Identifying footprints of selection in stocked brown trout populations: a spatio-temporal approach. Molecular Ecology 19: 1787-1800.

Hohenlohe, P. A., S. Bassham, P. D. Etter, N. Stiffler, E. A. Johnson et al., 2010 Population genomics of parallel adaptation in threespine stickleback using sequenced RAD tags. PLoS Genetics 6: e1000862.

Hornoy, B., N. Pavy, S. Gérardi, J. Beaulieu and J. Bousquet, 2015 Genetic adaptation to climate in white spruce involves small to moderate allele frequency shifts in functionally diverse genes. Genome Biology and Evolution 7: 3269-3285.

Huang, D. W., B. T. Sherman and R. A. Lempicki, 2009 Systematic and integrative analysis of large gene lists using DAVID bioinformatics resources. Nature Protocols 4: 44-57.

Kanehisa, M., and S. Goto, 2000 KEGG: kyoto encyclopedia of genes and genomes. Nucleic

Lande, R., 1988 Genetics and demography in biological conservation. Science 241: 14551460. 
645 Langella, O., 1999 POPULATIONS 1.2.30 Population genetic software. CNRS

646 UPR9034.[Online.] Available at bioinformatics. org/ tryphon/populations.

647 Larson, W. A., L. W. Seeb, M. V. Everett, R. K. Waples, W. D. Templin et al., 2014

648 Genotyping by sequencing resolves shallow population structure to inform

649 conservation of Chinook salmon (Oncorhynchus tshawytscha). Evolutionary

$650 \quad$ Applications 7: 355-369.

651 Lembi, C. A., B. G. Ritenour, E. M. Iverson and E. C. Forss, 1978 The effects of vegetation

652 removal by grass carp on water chemistry and phytoplankton in Indiana ponds.

653 Transactions of the American Fisheries Society 107: 161-171.

654 Li, H., and R. Durbin, 2010 Fast and accurate long-read alignment with Burrows-Wheeler

655 transform. Bioinformatics 26: 589-595.

656 Li, S., and F. Fang, 1990 Geographical distribution of the four kinds of pond-cultured carps.

$657 \quad$ Acta Zoologica Sinica 36: 244-250.

658 Liu, F., J. H. Xia, Z. Y. Bai, J. J. Fu, J. L. Li et al., 2009 High genetic diversity and substantial population differentiation in grass carp (Ctenopharyngodon idella) revealed by microsatellite analysis. Aquaculture 297: 51-56.

Liu, Z., and S. Li, 2015 China Fisheries Yearbook 2014. China Agriculture Press, Beijing, China.

McKay, J. K., and R. G. Latta, 2002 Adaptive population divergence: markers, QTL and traits. Trends in Ecology \& Evolution 17: 285-291.

McKown, A. D., R. D. Guy, J. Klápště, A. Geraldes, M. Friedmann et al., 2014 Geographical and environmental gradients shape phenotypic trait variation and genetic structure in Populus trichocarpa. New Phytologist 201: 1263-1276. quantitative traits. Journal of Evolutionary Biology 14: 892-903. 
670 Mitchell, C., 1986 Effects of introduced grass carp on populations of two species of small native fishes in a small lake. New Zealand Journal of Marine and Freshwater Research 20: 219-230.

Mitchell, S. E., E. S. Rogers, T. J. Little and A. F. Read, 2005 Host-parasite and genotype-by-environment interactions: temperature modifies potential for selection by Principal components analysis corrects for stratification in genome-wide association studies. Nature Genetics 38: 904-909. 
695 Raj, A., M. Stephens and J. K. Pritchard, 2014 fastSTRUCTURE: variational inference of

696 population structure in large SNP data sets. Genetics 197: 573-589.

697

698

699

Raymond, M., and F. Rousset, 1995 GENEPOP (version 1.2): population genetics software for exact tests and ecumenicism. Journal of Heredity 86: 248-249.

Reynolds, J., B. S. Weir and C. C. Cockerham, 1983 Estimation of the coancestry coefficient: basis for a short-term genetic distance. Genetics 105: 767-779.

Savolainen, O., S. T. Kujala, C. Sokol, T. Pyhäjärvi, K. Avia et al., 2011 Adaptive potential of northernmost tree populations to climate change, with emphasis on Scots pine (Pinus sylvestris L.). Journal of Heredity 102: 526-536.

Savolainen, O., M. Lascoux and J. Merilä, 2013 Ecological genomics of local adaptation. Nature Reviews Genetics 14: 807-820.

Seth, R. B., L. Sun, C. K. Ea and Z. J. Chen, 2005 Identification and characterization of MAVS, a mitochondrial antiviral signaling protein that activates NF- $\kappa$ B and IRF3. Cell 122: 669-682.

Sezgin, E., D. D. Duvernell, L. M. Matzkin, Y. Duan, C.-T. Zhu et al., 2004 Single-locus latitudinal clines and their relationship to temperate adaptation in metabolic genes and derived alleles in Drosophila melanogaster. Genetics 168: 923-931.

Shireman, J. V., and C. R. Smith, 1983 Synopsis of biological data on the grass carp, Ctenopharyngodon idella (Cuvier and Valenciennes, 1844). Food \& Agriculture Organization. Rome, Italy.

Skelton, P. H., 2001 A complete guide to the freshwater fishes of southern Africa. Southern Book Publishers, Halfway House, South Africa.

Stanley, J. G., 1976 Production of hybrid, androgenetic, and gynogenetic grass carp and carp. Transactions of the American Fisheries Society 105: 10-16. 
719 Storz, J. F., 2002 Contrasting patterns of divergence in quantitative traits and neutral DNA markers: analysis of clinal variation. Molecular Ecology 11: 2537-2551.

Sultan, S. E., and H. G. Spencer, 2002 Metapopulation structure favors plasticity over local adaptation. The American Naturalist 160: 271-283.

Vasemägi, A., 2006 The adaptive hypothesis of clinal variation revisited: single-locus clines as a result of spatially restricted gene flow. Genetics 173: 2411-2414.

725 Wang, L., S. Liu, Z. Zhuang, L. Guo, Z. Meng et al., 2013 Population genetic studies revealed local adaptation in a high gene-flow marine fish, the small yellow croaker Agriculture Organisation of the United Nations. Rome, Italy. (Larimichthys polyactis). PLoS ONE 8: e83493.

Wang, L., Z. Y. Wan, H. S. Lim and G. H. Yue, 2015a Genetic heterogeneity and local adaptation of Asian seabass across Indonesian Archipelago revealed with geneassociated SNP markers. Fisheries Research 170: 205-211.

Wang, Y., Y. Lu, Y. Zhang, Z. Ning, Y. Li et al., 2015b The draft genome of the grass carp (Ctenopharyngodon idellus) provides insights into its evolution and vegetarian adaptation. Nature Genetics 47: 625-631.

Ward, R., M. Woodwark and D. Skibinski, 1994 A comparison of genetic diversity levels in marine, freshwater, and anadromous fishes. Journal of Fish Biology 44: 213-232.

Weir, B. S., and C. C. Cockerham, 1984 Estimating F-statistics for the analysis of population structure. Evolution 38: 1358-1370.

Welcomme, R. L., 1988 International introductions of inland aquatic species. Food \&

Ye, J., L. Fang, H. Zheng, Y. Zhang, J. Chen et al., 2006 WEGO: a web tool for plotting GO annotations. Nucleic Acids Research 34: 293-297. 
742 Zhang, S., H. Deng, D. Wang and L. Yu, 2001 Population structure and genetic diversity of

743 silver carp and grass carp from populations of Yangtze river system revealed by

744 RAPD. Acta Hydrobiologica Sinica 25: 330-336.

745 Zhu, D., 1993 The Dictionary of River Systems in China. Qingdao Press, Qingdao, China. 


\section{Supporting information}

762 Table S1 Summary statistics of annotated genes under putative directional selection and their

763 potential functions in grass carp

764 Table S2 Enriched KEGG pathways and the candidate genes under potential selection in

765 grass carp

766

767

768

\section{Tables}

769 Table 1 Sampling information of six native and three introduced grass carp populations

770 including river systems of origin, numbers of samples, sampling localities and dates, and the

771 annual average temperature of each sampling locality. Measures of genetic diversity

772 including observed heterozygosity $\left(H_{O}\right)$, expected heterozygosity $\left(H_{E}\right)$ and nucleotide

773 diversity $(\Pi)$ are also indicated.

\begin{tabular}{llllllllll}
\hline Samples & Origin & $\mathrm{N}$ & Longitude & Latitude & Date & Temperature & $\mathrm{H}_{\mathrm{O}}$ & $\mathrm{H}_{\mathrm{E}}$ & $\Pi$ \\
\hline Nenjiang & Heilongjiang River System & 22 & 125.22 & 49.21 & 2007 & 4.3 & 0.197 & 0.202 & 0.207 \\
Hanjiang & Yangtze River System & 26 & 119.43 & 32.35 & 2007 & 15.4 & 0.200 & 0.201 & 0.205 \\
Jiujiang & Yangtze River System & 23 & 115.96 & 29.72 & 2007 & 17.7 & 0.202 & 0.205 & 0.210 \\
Shishou & Yangtze River System & 11 & 112.39 & 29.74 & 2007 & 16.9 & 0.210 & 0.204 & 0.214 \\
Zhaoqing & Pearl River System & 21 & 112.53 & 23.08 & 2007 & 22.7 & 0.203 & 0.201 & 0.206 \\
Vietnam & Pearl River System & 26 & 105.98 & 21.12 & 2008 & 24.8 & 0.190 & 0.191 & 0.195 \\
Malaysia & Introduced & 18 & 101.15 & 4.58 & 2008 & $\mathrm{Na}$. & 0.136 & 0.126 & 0.130 \\
India & Introduced & 25 & 83.37 & 26.76 & 2008 & $\mathrm{Na}$. & 0.171 & 0.168 & 0.171 \\
Nepal & Introduced & 25 & 85.03 & 27.42 & 2008 & $\mathrm{Na}$. & 0.161 & 0.152 & 0.155
\end{tabular}

774 
776 Table 2 Pairwise $F_{S T}$ values among each pair of populations of grass carp. Genetic

777 differentiation that was non-significant after Bonferroni corrections $(P<0.001)$ is denoted in

778 bold.

\begin{tabular}{|c|c|c|c|c|c|c|c|c|c|}
\hline & Nenjiang & Hanjiang & Jiujiang & Shishou & Zhaoqing & Vietnam & India & Nepal & Malaysia \\
\hline Nenjiang & - & & & & & & & & \\
\hline Hanjiang & 0.0260 & - & & & & & & & \\
\hline Jiujiang & 0.0228 & 0.0154 & - & & & & & & \\
\hline Shishou & 0.0196 & 0.0123 & 0.0073 & - & & & & & \\
\hline Zhaoqing & 0.0272 & 0.0351 & 0.0337 & 0.0279 & - & & & & \\
\hline Vietnam & 0.0448 & 0.0515 & 0.0484 & 0.0449 & 0.0238 & - & & & \\
\hline India & 0.1265 & 0.1307 & 0.1282 & 0.1289 & 0.1126 & 0.1297 & - & & \\
\hline Nepal & 0.1540 & 0.1580 & 0.1532 & 0.1596 & 0.1384 & 0.1592 & 0.1814 & - & \\
\hline Malaysia & 0.2295 & 0.2255 & 0.2224 & 0.2336 & 0.2205 & 0.2399 & 0.3167 & 0.3501 & - \\
\hline
\end{tabular}

779

780

781

782

783

784

785

786

787

788

789 
790 Table 3 Enriched candidate genes under putative directional selection and their potential

791 functions in grass carp. Approaches, including $F_{S T}$ outlier tests, Bayenv and allele frequency

792 correlations (AFC), that were used to determine if one gene was under putative selection, are

793 also indicated.

\begin{tabular}{|c|c|c|c|c|c|}
\hline Locus & Gene name & Function & \multicolumn{3}{|c|}{ Approaches } \\
\hline Lca7796 & Toll-like receptor $5 \mathrm{~b}$ & Immune response & $F_{S T}$ outlier & & \\
\hline Lca7927 & Chemokine (C-C motif)-like & Immune response & & & AFC \\
\hline Lca15228 & DEXH (Asp-Glu-X-His) box polypeptide 58 & Antiviral signaling & $F_{S T}$ outlier & & \\
\hline Lca33879 & Phosphofurin acidic cluster sorting protein 2 & Antiviral signaling & & Bayenv & \\
\hline Lca52319 & Myxovirus (influenza virus) resistance $\mathrm{E}$ & Antiviral signaling & $F_{S T}$ outlier & Bayenv & AFC \\
\hline Lca57825 & Integrin alpha FG-GAP repeat containing 1 & Antiviral signaling & & Bayenv & $\mathrm{AFC}$ \\
\hline Lca79226 & Interleukin-1 receptor-associated kinase 1 & Immune response & $F_{S T}$ outlier & & \\
\hline Lca88973 & Major histocompatibility complex class I antigen UKA & Immune response & $F_{S T}$ outlier & Bayenv & AFC \\
\hline Lca1 10035 & Grass carp reovirus (GCRV)-induced gene 21 & Antiviral signaling & $F_{S T}$ outlier & & \\
\hline Lca152085 & Interferon induced with helicase $\mathrm{C}$ domain 1 & Immune response & $F_{S T}$ outlier & & \\
\hline Lca152284 & PC4 and SFRS1 interacting protein 1 & Antiviral signaling & $F_{S T}$ outlier & & $\mathrm{AFC}$ \\
\hline Lca159757 & Major histocompatibility complex class II DAB & Immune response & $F_{S T}$ outlier & & \\
\hline Lca175136 & Lymphocyte cytosolic protein 1 & Immune response & & & $\mathrm{AFC}$ \\
\hline Lca183026 & Myxovirus (influenza) resistance A & Antiviral signaling & $F_{S T}$ outlier & & \\
\hline Lca202544 & Baculoviral IAP repeat containing 2 & Immune response & $F_{S T}$ outlier & & \\
\hline Lca285612 & Mitochondrial antiviral signalling protein & Antiviral signaling & & & AFC \\
\hline
\end{tabular}

794

795

796

797

798

799 


\section{Figures}

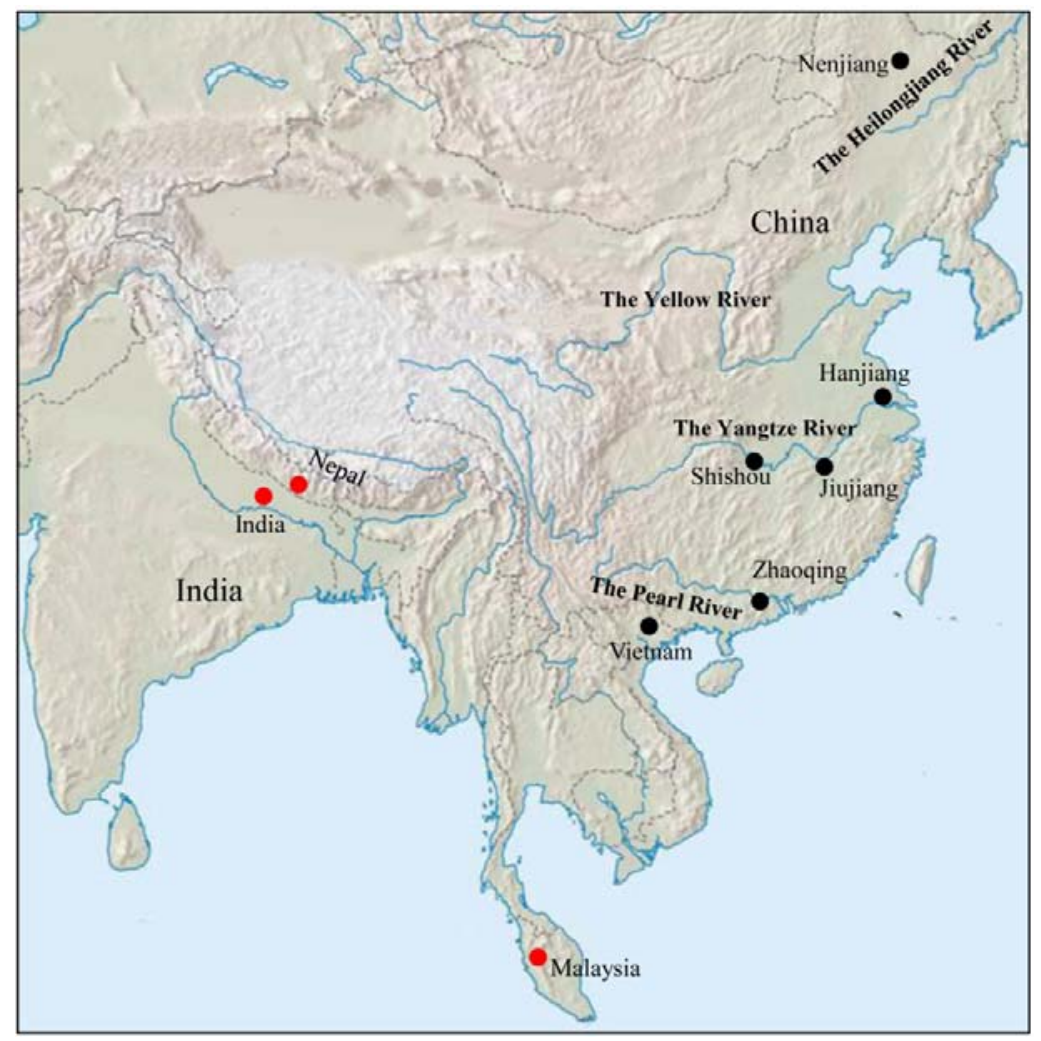

802 Figure 1 Sampling sites of six native grass carp populations distributed in the three river systems: the Heilongjiang River, the Yangtze River and the Pearl River Systems, and three introduced populations from Malaysia, India and Nepal. The wild and introduced populations are denoted as black and red solid circles, respectively. Detailed sampling information is listed in Table 1. 


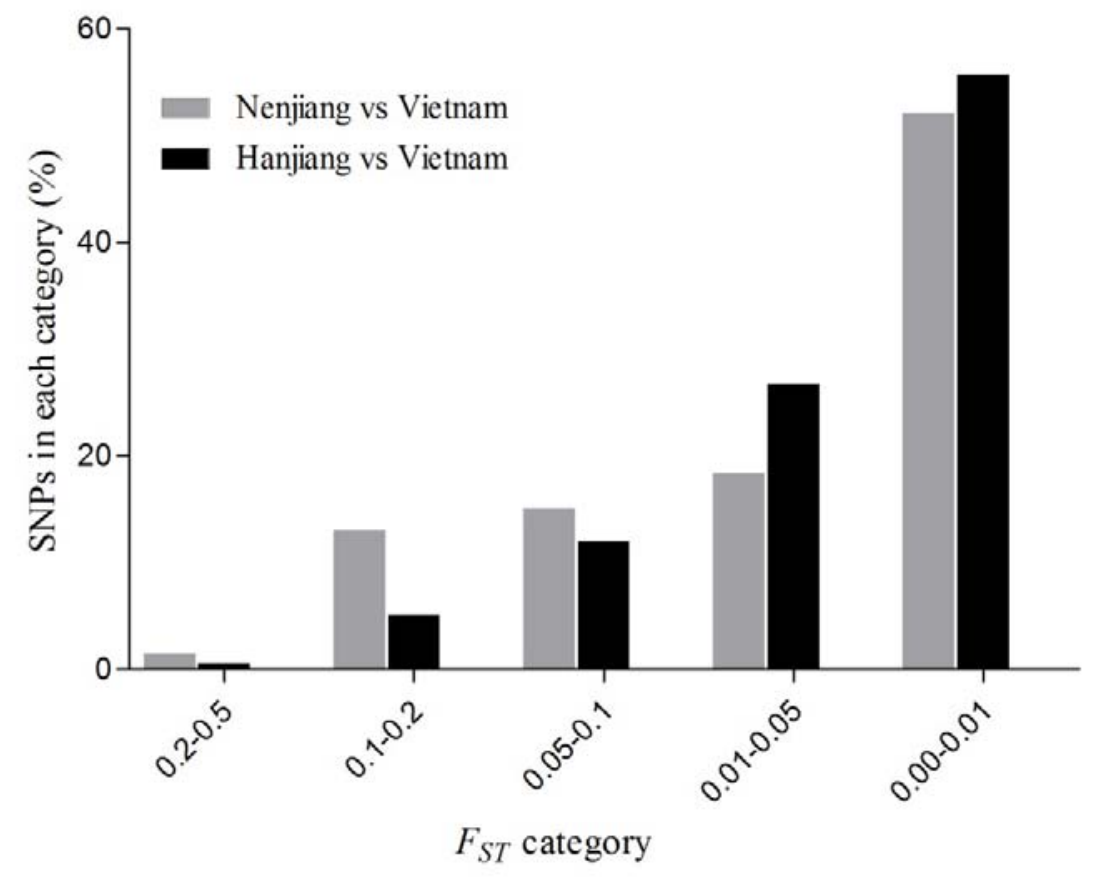

813 Figure 2 Distribution of $F_{S T}$ values in different categories between Nenjiang and Vietnam,

814 with the longest geographical distance, and between Hanjiang and Vietnam, with the largest

815 genetic distance, based on all genotyped SNPs with MAF $>0.05$. 


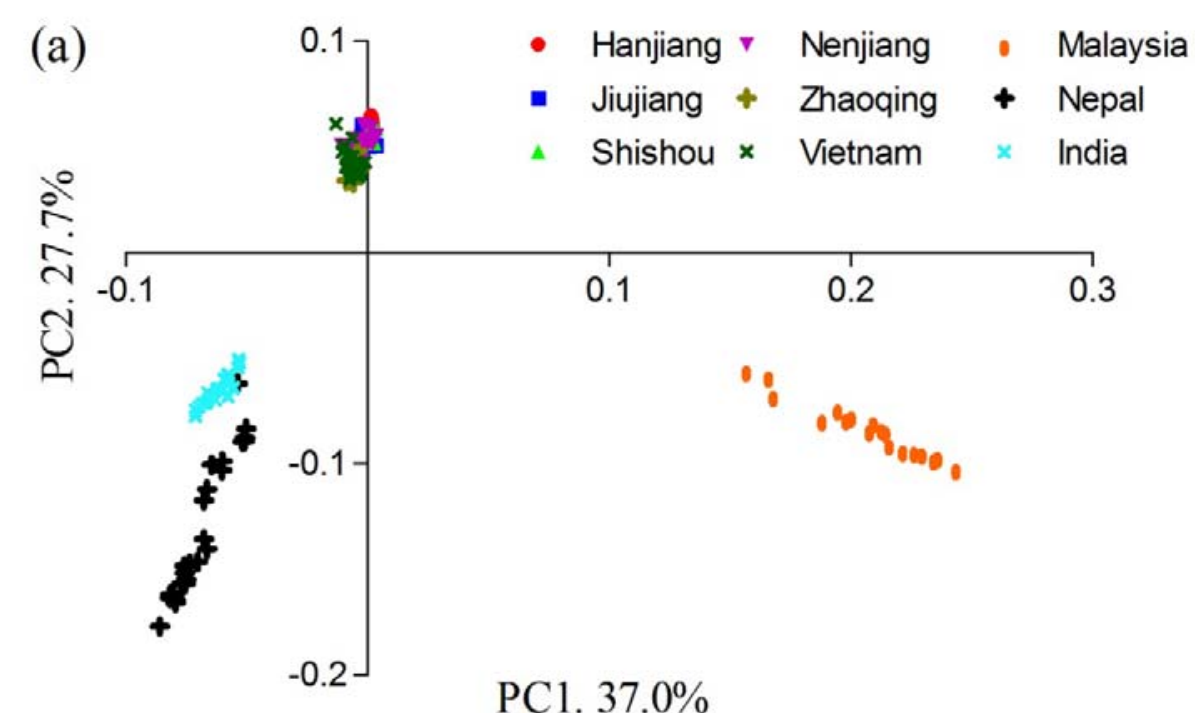

824

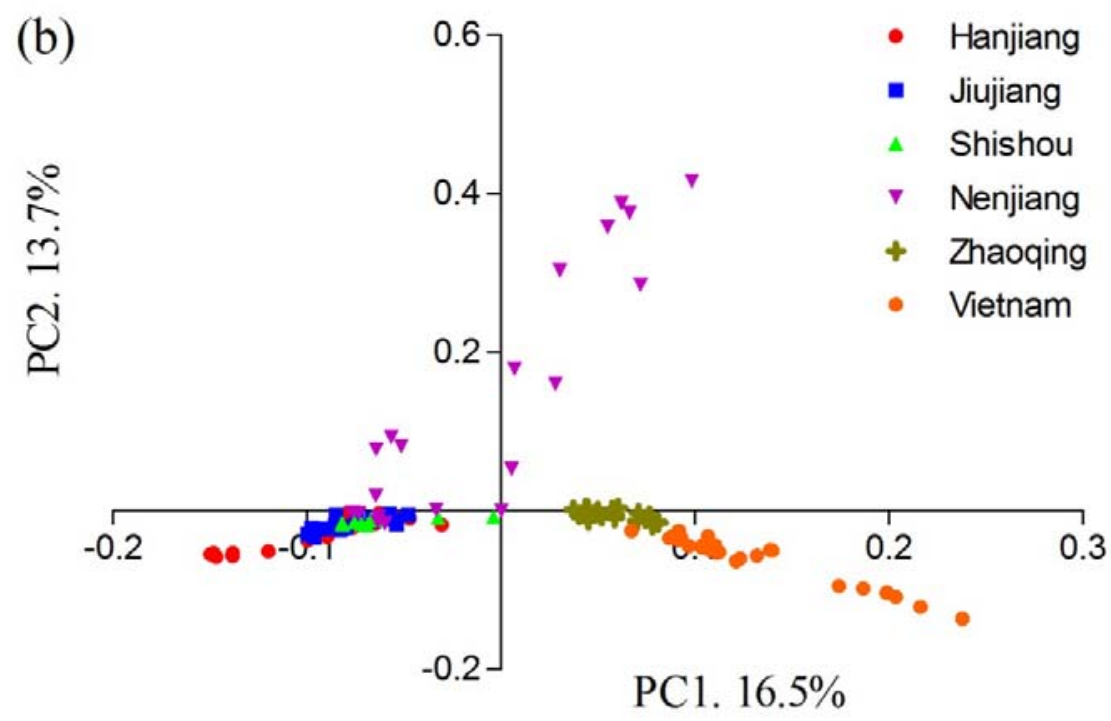

825

826 Figure 3 Principal component analyses for (a) all nine populations and (b) six native

827 populations of grass carp based on all genotyped SNPs. 
831

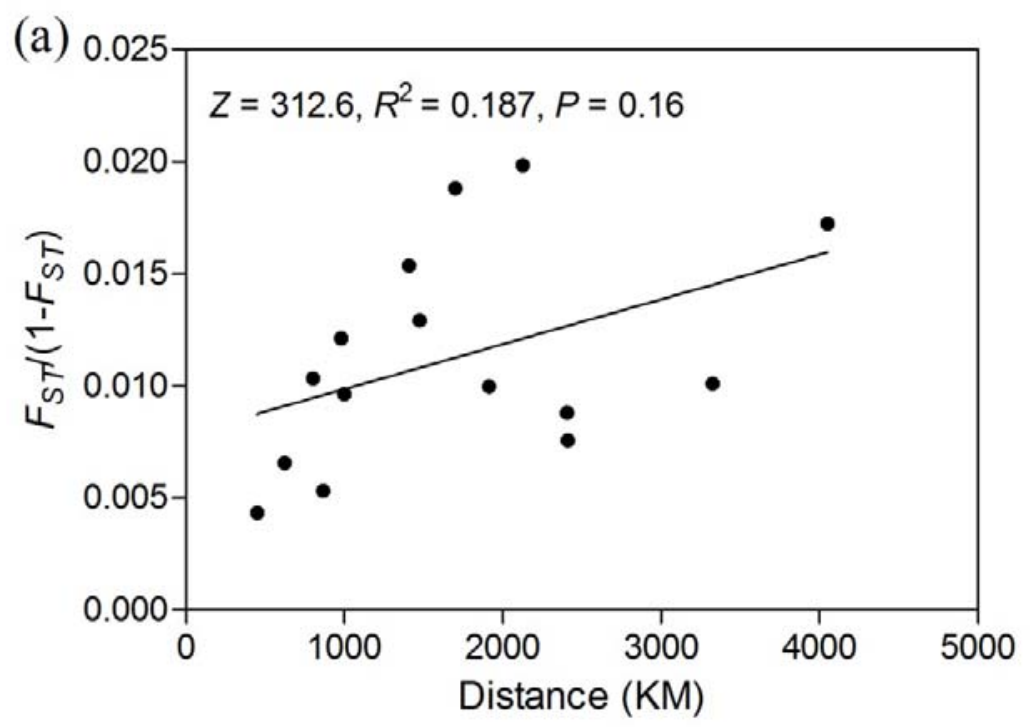

832

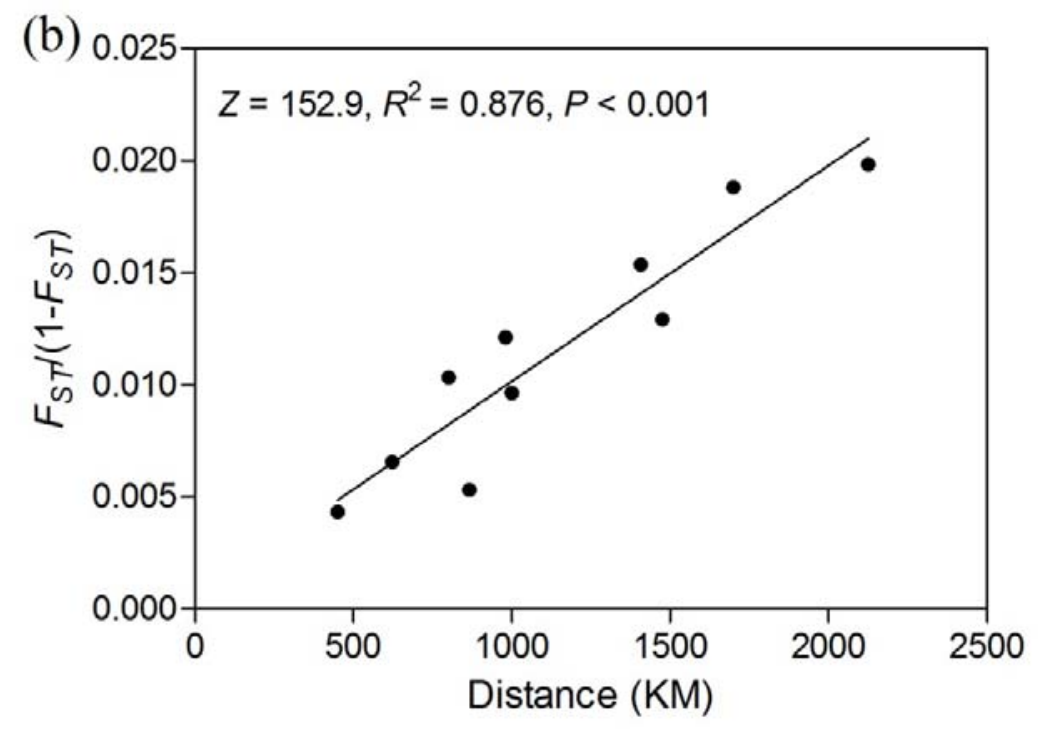

833 Figure 4 The overall pattern of isolation-by-distance for (a) all six native populations and (b)

834 five native populations excluding population Nenjiang, examined using Mantel tests based on

835 all genotyped SNPs. Genetic distance was estimated as $F_{S T} /\left(1-F_{S T}\right)$, while geographical

836 distance was the linear distance between sampling localities. 
(a)

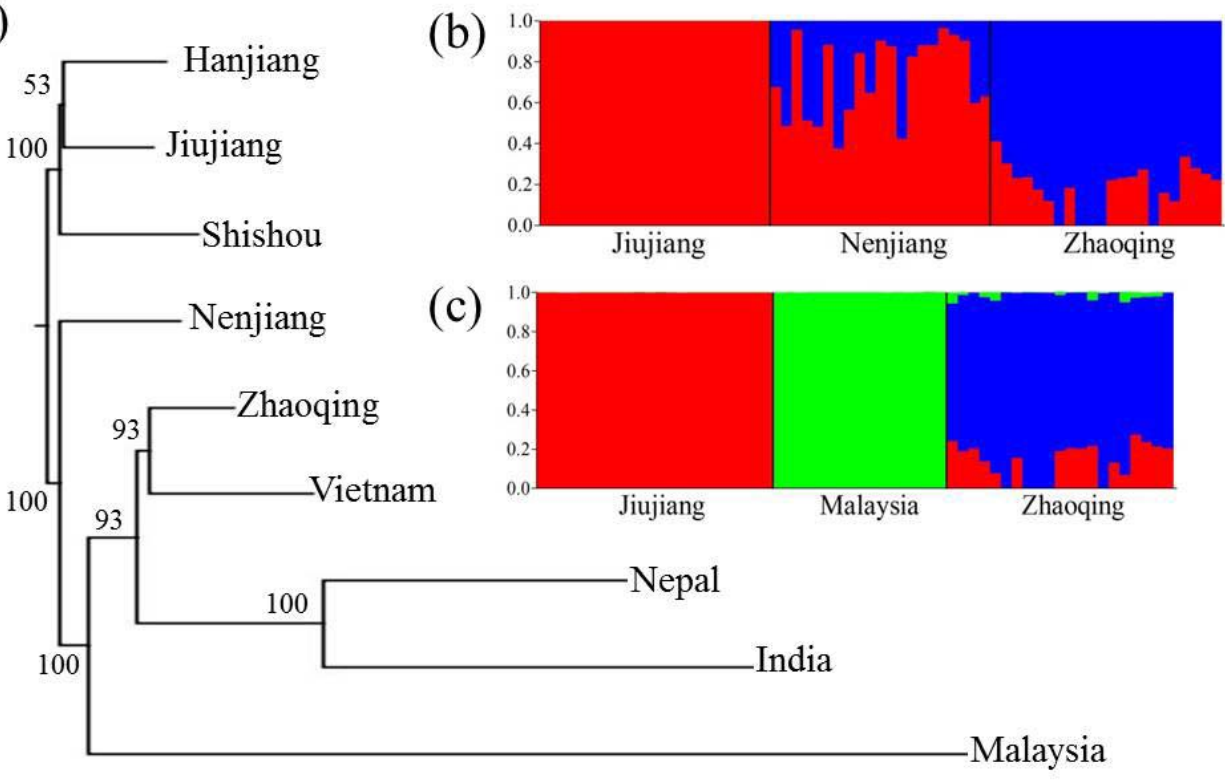

840

841 Figure 5 (a) Phylogenetic relationships among all nine populations of grass carp constructed 842 using the Neighbor-Joining approach. Bootstrap supports over loci for 1000 times are 843 indicated. (b) Genetic assignment of the native population Nenjiang to the Yangtze River 844 System and the Pearl River System and (c) genetic assignment of the introduced population 845 Malaysia to the Yangtze River System and the Pearl River System, respectively. The most 846 likely $\mathrm{K}$ value for both assignment tests in the program Structure was inferred as 5. Each 847 vertical line represents one individual, while each colour shows the genetic composition that 848 is assigned into a distinct genetic cluster. 


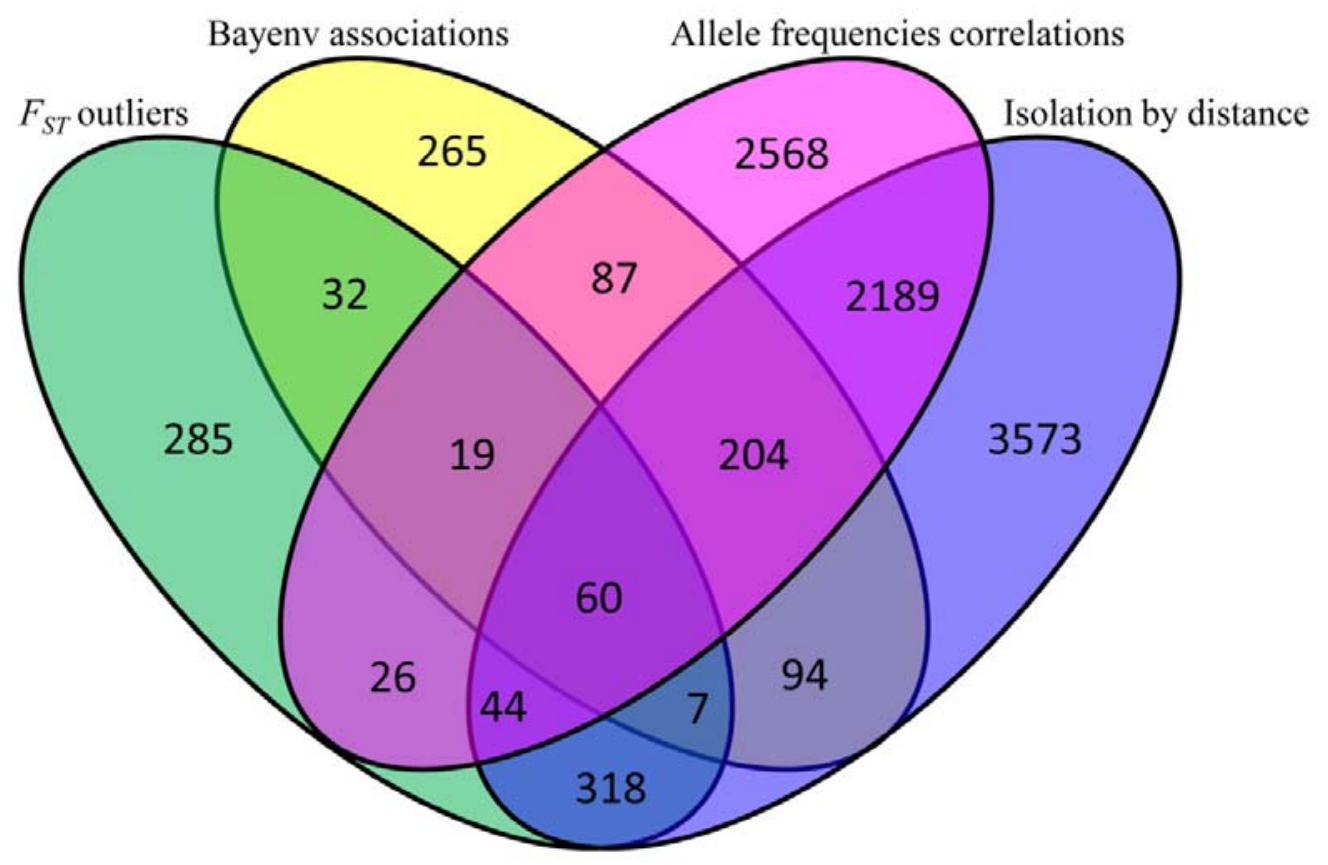

854 Figure 6 Venn diagrams showing the number of loci under putative selection and isolation-

855 by-distance as revealed by $F_{S T}$ outlier tests, Bayenv association tests, Allele frequency

856 correlations and Mantels tests for Isolation-by-distance. The numbers of overlapping loci

857 among the different approaches are also illustrated. 


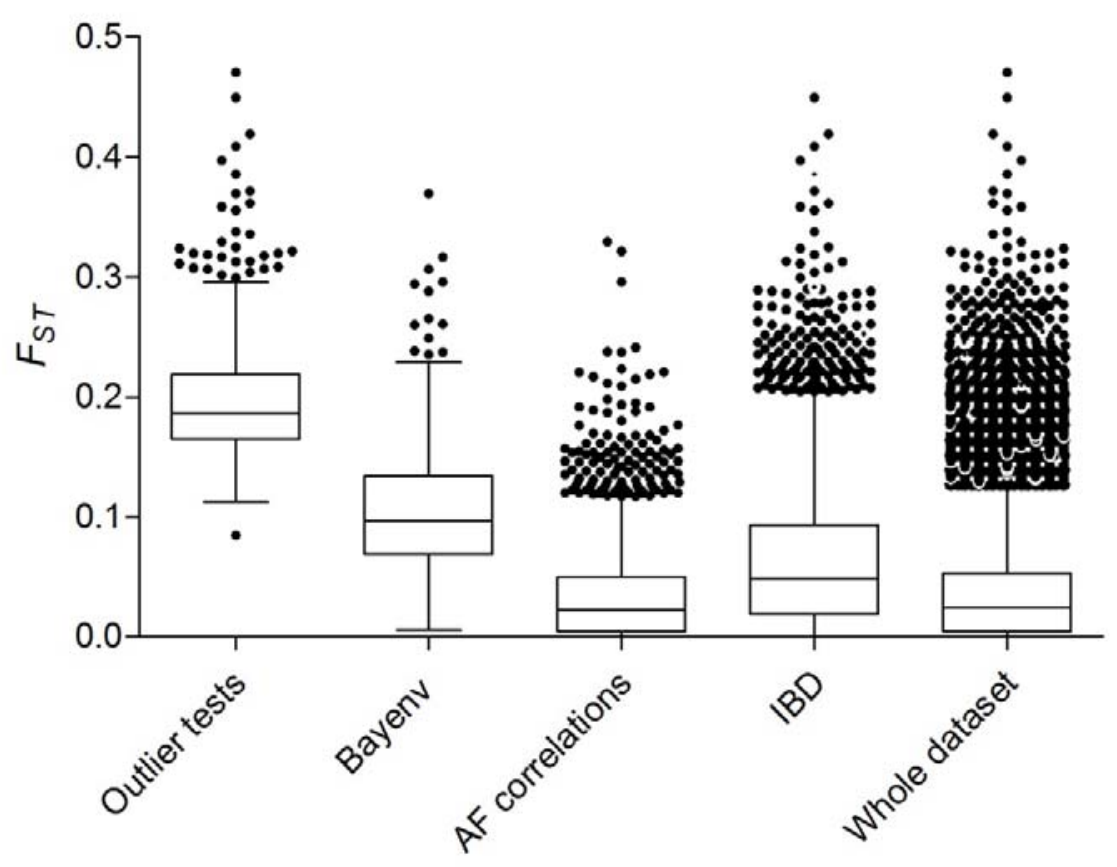

867 Figure 7 Distribution of $F_{S T}$ values for the SNPs that were identified as outliers for putative

868 directional selection, associated with latitude as revealed by Bayenv, had allele frequencies

869 correlated to latitude and agreed with the pattern of isolation-by-distance, as well as for the

870 whole dataset. The highest and lowest error bar indicates the 95\% quantile, while the median

871 horizontal line denotes the mean $F_{S T}$ value. Individual locus with $\mathrm{F}_{\mathrm{ST}}$ over the upper $95 \%$

872 quantile is shown. 


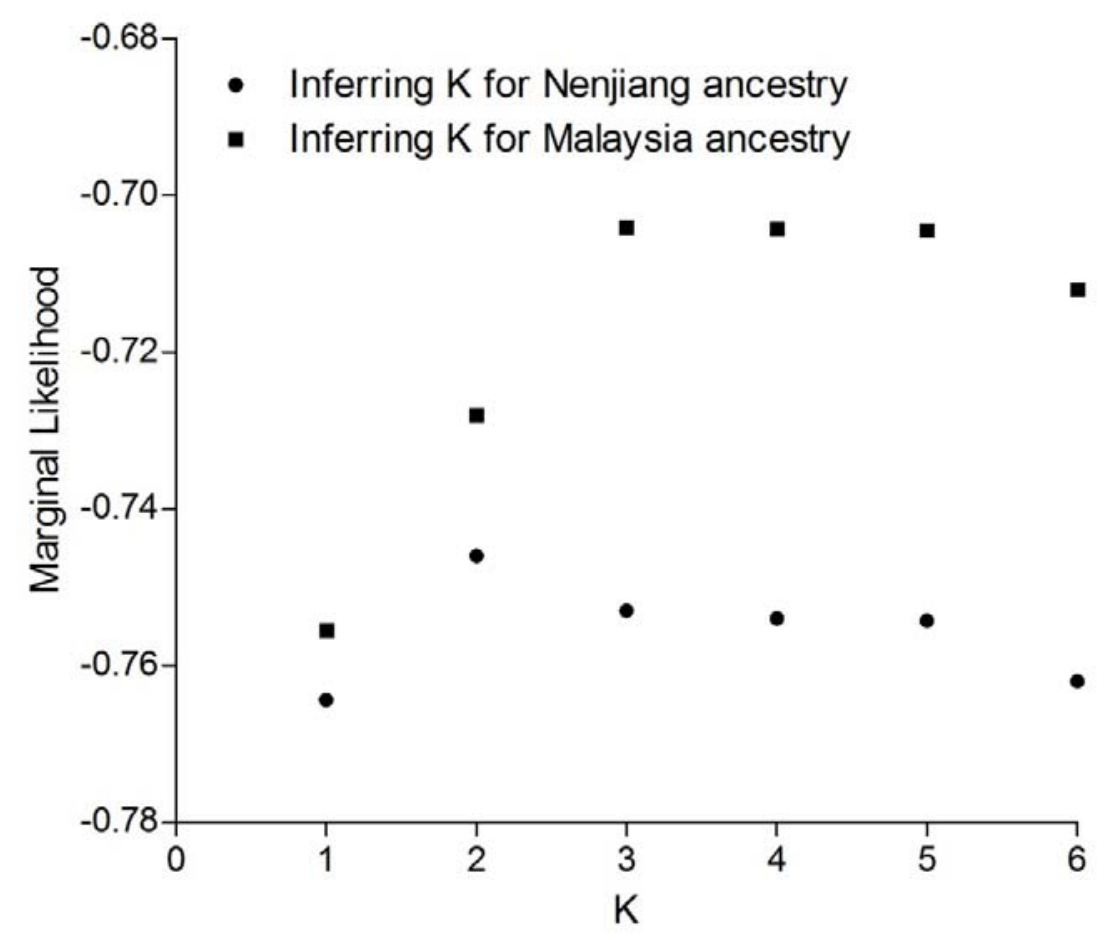

879

880 Figure S1 Plotting of K values inferred from the program fastStructure.

881

882

883

884

885

886

887

888

889 


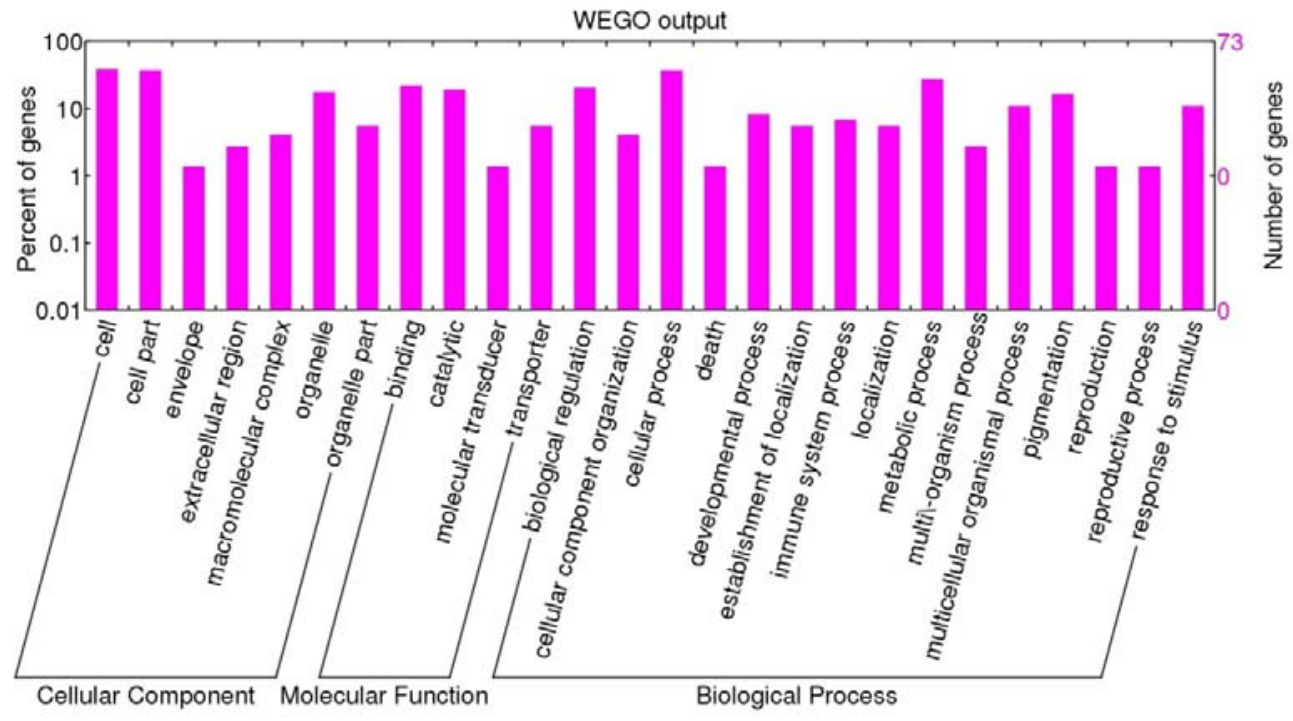

890

891 Figure S2 Gene ontology annotations of the candidate genes under potential local selection

892 in grass carp. Three categories: Cellular Component, Molecular Function and Biological

893 Process, were used to visualize the potential functions of enriched genes 